\title{
Is Wound Complication a Deterrent to Lateral Extensile Approach in Calcaneus Fracture?
}

\author{
Leow Voon Chin, Lee Jie Ying, Mohamad Izani \\ Hospital Raja Permaisuri Bainun, Ipoh, Malaysia \\ Email:voon325@yahoo.com
}

How to cite this paper: Chin, L.V., Ying, L.J. and Izani, M. (2019) Is Wound Complication a Deterrent to Lateral Extensile Approach in Calcaneus Fracture? Open Journal of Orthopedics, 9, 180-189. https://doi.org/10.4236/ojo.2019.98019

Received: July 8, 2019

Accepted: August 10, 2019

Published: August 13, 2019

Copyright $\odot 2019$ by author(s) and Scientific Research Publishing Inc. This work is licensed under the Creative Commons Attribution International License (CC BY 4.0).

http://creativecommons.org/licenses/by/4.0/

cc) (i) Open Access

\begin{abstract}
Introduction: The lateral extensile approach has been a widely accepted surgical approach in treatment of intraarticular calcaneal fracture. It provides good exposure for fixation by correcting the hindfoot varus, fracture reduction and restores calcaneal height but it has a high wound complication rate. Methods and materials: 36 intraarticular calcaneal fractures (Sanders II, III and IV) treated using the lateral extensile approach in 34 patients presenting between 2015 and 2018 were retrospectively reviewed. Wound complication in the early stage (day 1 to day 3), intermediate (2 weeks) and late (6 weeks) were documented. Results: We found early (Day 1 - Day 3 ) infection rate of $5.6 \%$, intermediate ( 2 weeks) at $17.6 \%$ and late wound complication (6 weeks) at $2.9 \%$. Conclusion: Lateral expansile approach is a reliable surgical exposure for fracture reduction of calcaneal fractures with acceptable wound related complication rate. Minimally invasive techniques and the sinus tarsi approach are being used for less comminuted fractures, but those techniques are technically more demanding. Smoking, long duration of surgery and soft tissue handling also play a role in increasing wound complication rates.
\end{abstract}

\section{Keywords}

Wound Complications, Calcaneal Fracture, Lateral Extensile Approach

\section{Introduction}

Calcaneum fracture accounts for approximately $2 \%$ of all fractures. We treated 60 cases of calcaneum fracture in our center from 2015 to 2018. Majority of the cases were treated operatively due to intraarticular involvement, calcaneal tuberosity avulsion fracture and open fracture of the calcaneum (Figure 1).

Early reports on non-operative displaced calcaneal fractures reflected poor functional outcome due to unreduced intraarticular fracture of the posterior facet, 


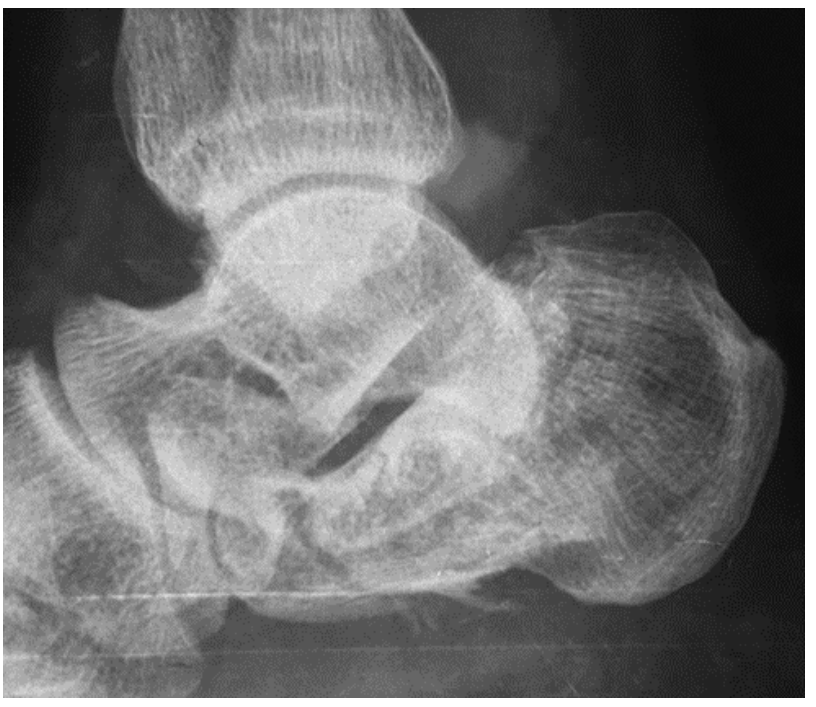

Figure 1. Typical intraarticular calcaneal fracture at our center.

reduced in calcaneal height, blow-out of lateral wall causing peroneal tendon irritation, increased width of the calcaneum and varus deformity of the hindfoot [1].

Choosing a non-operative treatment can avoid wound complications result in many late complications such as hindfoot malalignment, peroneal tendon dislocation, subtalar joint dysfunction and foot mechanics disruption [2].

Open reduction and internal fixation with calcaneal plate (conventional or locking) via a lateral extensile approach have been considered a standard treatment for displaced intraarticular calcaneal fractures because it provides excellent exposure of the fracture site. It also allows direct reduction of the depressed posterior facet fragment, addresses the blow-out lateral wall and corrects the malalignment tuberosity. However, wound complication such as wound edge necrosis, dehiscence, hematoma, or deep infection has been reported high in several studies. Koski et al., Al-Mudhaffar et al. and Vaclav et al. reported an overall wound complication rate for lateral extensile approach of $24 \%, 18.1 \%$ and $17 \%$ respectively [3] [4] [5].

We present our current data with regards to wound complications associated with the lateral extensile approach, to assess its feasibility in treatment of calcaneum fracture fixation.

\section{Materials and Methods}

This is a retrospective study involving all patients with calcaneum fracture that were treated with lateral extensile approach for fracture fixation of the calcaneum from January 2015 to December 2018. All the cases were performed by the orthopedic foot and ankle surgeon and his fellow (with supervision) at our center.

The presence of skin edges necrosis, wound breakdown or dehiscence with or without pus discharge and surgical site redness or cellulitis during wound in- 
spection days was recorded as a complication.

Documentation of wound inspection was done on wound inspection day (post-operative day 1 to day 3), at 2 weeks and 6 weeks for final wound healing.

Related factors which may influence the wound outcome such as age, timing of surgery, Sanders Classification for calcaneum fracture, duration of surgery in minutes, suction drainage post-surgery, presence of concomitant fractures, patient's diabetic status and smoking habit were also analyzed.

The operative approach was done with the patient in the lateral decubitus position.

The surgical foot area was cleaned first with chlorohexidine then followed by povidone iodine and draped under sterile technique. The forefoot not involved in the surgical exposure was covered with a transparent adhesive layer. A standard extensile lateral approach was used to raise a full thickness skin flap off the lateral calcaneal wall. A gentle curve cut or angle cut with a size 15 blade (Figure 2 ) avoids oblique skin edges which are crucial in the early part of wound healing. The full thickness flap was raised slowly with blade size 10 using the suture retraction technique (Figure 3) minimizing usage of Senmilar retractors. Electrical cautery was not used to raise the flap. 3 to $41.8 \mathrm{~mm}$ Kirshner wires served as static retraction poles at the cuboid, anterior and posterior aspect of talus to maintain the skin flap during bone reduction work. This will allow adequate visualization of the fractured posterior facet.

A suction drain to prevent post-operative hematoma formation was inserted prior to closure (Figure 4). The subcutaneous layer was closed with absorbable $3 / 0$ and skin with nylon 3/0 and tight compression dressing was avoided to reduce post-operative pain and discomfort.

Wound inspection was done on day lif the outer layer dressing was soaked. Otherwise it was inspected on day 3.

All early wound complications will be kept for intravenous antibiotics and wound dressing. Patients were reviewed at 2 weeks and 6 weeks, or earlier depending on their wound condition.

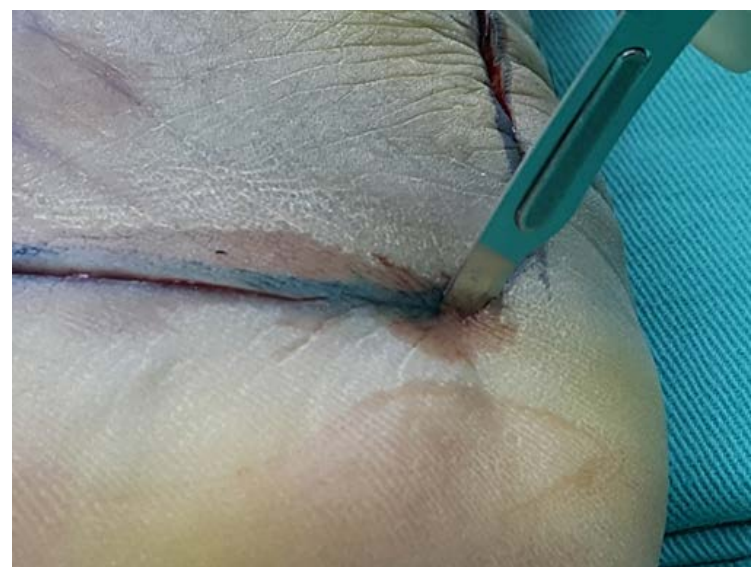

Figure 2. Blade size 15 was used for the curve linear incision to ensure straight to bone full thickness flap. 


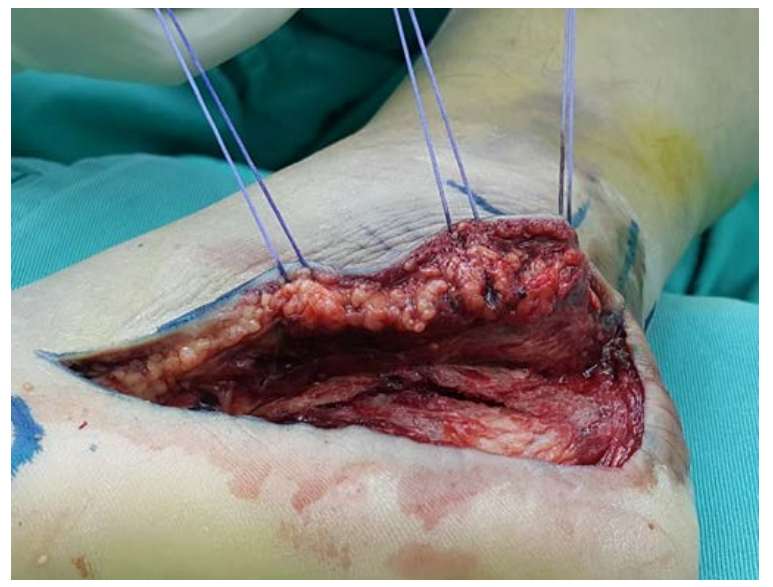

Figure 3. Our own suture retraction technique to raise a full thickness flap to ensure minimum trauma during retraction.

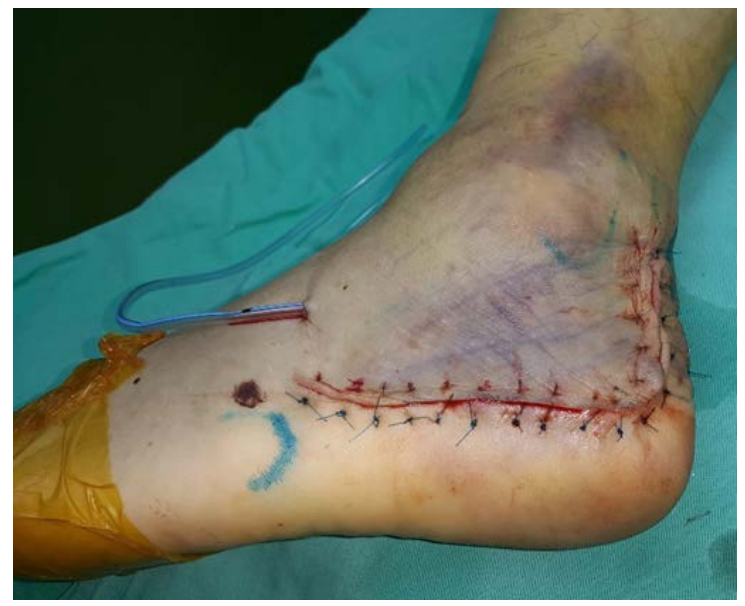

Figure 4. Closing mattress suture without tension and insertion of a suction drain.

All patients' data collection from their medical records are consented and we obtained approval from Clinical Research Centre and National Medical Research Register Malaysia (Research ID 44279).

Statistical analysis was done using IBM SPSS Statistics 23.0. Independent $\mathrm{t}$-test and Fisher's exact test were used.

\section{Results}

There were 36 calcaneum fractures in 34 patients treated with lateral extensile approach in this study. 30 out of the 34 patients were male and majority of them were industrial workers who sustained the fracture from motor vehicle accident or fall from height at the construction site. Their age ranged from 20 to 71 years old with the mean age of 36.6 years.

26 patients had isolated calcaneal fracture while 3 patients ( 6 cases) had bilateral calcaneal fracture that was treated with the lateral extensile approach. 2 patients had a concomitant fracture lumbar burst fracture, 1 had bilateral distal end radius fracture and another had a contralateral tibia plateau fracture, which 
were all treated operatively.

Out of the 36 cases, 34 had computed tomography (CT) scan of the calcaneum. 19 were Sanders II, 13 Sanders III, 2 Sanders IV while another 2 were inconclusive, but taken to be Sanders II or III based on the intraoperative findings. All the cases had a disrupted Bohler angle.

The timing for surgery was decided by the operating surgeon. All the cases were assessed for the presence of wrinkle sign before proceeding for surgery. The day of injury to day of surgery ranged from 2 to 20 days, with a mean of 9.2 days.

The operating time was 75 to 185 minutes with the meantime 112.9 minutes.

All the cases had a suction drain inserted. The amount of blood drained ranged from $10 \mathrm{cc}$ to $650 \mathrm{cc}$, with a mean amount was $142.7 \mathrm{cc}$. We removed the drainage tube on day 2 or day 3 depending if drainage was less than $30 \mathrm{cc}-50 \mathrm{cc}$ and the operated foot was less tense.

2 out of 36 cases (5.6\%) had early wound complications when we inspected the wound within the first 3 days after surgery. In the 2 cases, 1 with wound edges necrosis and another with haemoserous discharge. The patient with serous discharge had daily dressing and wound healed after 2 weeks. The other patient (patient $\mathrm{M}$ ) had wound edge necrosis and continued to have wound dehiscence and serous discharge. He underwent surgical debridement.

At the 2 weeks outpatient clinic follow-up, only 34 cases were available for review. We found 6 out of 34 (17.6\%) had surgical site wound related complication, including patient $(\mathrm{M}) .4$ out of 6 had serous discharge and wound dehiscence. 2 out of the 6 has no wound breakdown but instead treated as cellulitis due to warm and erythematous skin changes.

At 6 weeks, 5 of the cases ( 3 serous discharge, 2 redness) that was found to have complication at 2 weeks healed completely with no further wound complication. Patient $\mathrm{M}$ continued to have persistent infection, underwent multiple debridement and eventually hardware removal. That gave us the final complication rate (at 6 weeks) was 1 out of 34 cases, $2.9 \%$.

All the cases included in the study had no history of diabetes. 20 out of 36 were smoker (more than 10 sticks per day) and 16 were non-smokers.

Demographic characteristics of patient treated with lateral extensile approach are shown in Table 1 and the possible risk factor analysis is shown in Table 2.

Table 1. Demographic characteristics of patient treated with lateral extensile approach during calcaneal fracture surgery at Hospital Raja Permaisuri Bainun.

\begin{tabular}{cc}
\hline Variable & Frequency \\
\hline Mean Age (SD) & $36.6(11.5)$ \\
Gender & Male $=30$, Female $=4(\mathrm{n}=34)$ \\
Timing of surgery (days) & $9.2(4.6)$ \\
Sanders classification & Type 2 $=19$, Type 3 $=13$, Type $4=2(\mathrm{n}=34)^{*}$ \\
Duration of surgery (min) & $112.9(26.3)$ \\
Amount of blood in suction drain $(\mathrm{cc})$ & $142.7(119.4)$ \\
\hline
\end{tabular}




\section{Continued}

\begin{tabular}{cc}
\hline Wound complication Day 1 - Day 3 & 2 \\
Wound complication at 2 weeks & 4.2 (cellulitis) $=6$ \\
Wound complication at 6 weeks & 1 \\
Smoking status & No $=14$, Yes $=20(\mathrm{n}=34)$ \\
Diabetes status & 0 \\
\hline
\end{tabular}

*2 patients do not have CT scan.

Table 2. Risk factors of infection complication in lateral extensile approach for calcaneal fracture surgery, Hospital Raja Permaisuri Bainun.

\begin{tabular}{|c|c|c|c|c|}
\hline \multirow{2}{*}{\multicolumn{2}{|c|}{ Variable }} & Complication & Non-complication & \multirow{2}{*}{ p-value } \\
\hline & & Mean (SD) & Mean (SD) & \\
\hline Duration of & $(\min )$ & $123.8(19.31)$ & $109.7(23.95)$ & $0.272^{\mathrm{a}}$ \\
\hline \multirow{2}{*}{ Smoking* } & Yes & $4(-)$ & $15(-)$ & \multirow{2}{*}{$0.113^{\mathrm{b}}$} \\
\hline & No & $0(-)$ & $15(-)$ & \\
\hline \multicolumn{2}{|c|}{ Age (years) } & $38.3(10.34)$ & $36.4(11.78)$ & $0.763^{\mathrm{a}}$ \\
\hline \multicolumn{2}{|c|}{ Days to surgery (days) } & $10.3(3.95)$ & $9.3(4.68)$ & $0.702^{\mathrm{a}}$ \\
\hline
\end{tabular}

andependent t-test; ${ }^{*}$ presented with frequency; ${ }^{b}$ Fisher's Exact test.

\section{Discussion}

This retrospective study presents the wound complication rate in the operative treatment of intraarticular calcaneum fracture using the lateral extensile approach at our center. In our experience, this approach provides good visualization of the fracture and allows for fracture fixation with direct reduction [6] [7] [8].

We found the early surgical wound related complication rate of $17.6 \%$ at 2 weeks follow-up, which were 6 patients. However, 2 out of the 6 had no surgical wound breakdown, but just redness which resolved completely with oral antibiotics. 4 out of the 34 patients had surgical wound complications that presented with serous discharge and wound dehiscence. Only 1 from the 34 patients had deep infection required hardware removal. The rest of 33 patients, healed without further wound complication at 6 weeks.

All cases with surgical wound complication at 2 weeks (17.6\%) were operated 7 - 15 days after injury after the foot was assessed for the presence of wrinkle sign. The operative time ranged from $115 \mathrm{~min}$ to $150 \mathrm{~min}$. All of them were smokers, smoking more than 10 sticks per day.

We found our complication rate comparable to other reported studies. Al-Mudhaffar reported a wound complication rate of $18.1 \%$. [4]. A large series of 190 patients by Folk and colleague with lateral extensile approach reported a $25 \%$ rate of infection and $21 \%$ required surgical debridement [9]. Koski et al. also reported an almost similar rate at $24 \%$ [3]. Rak et al. reported a rather low complication rate of $17 \%$ in non-locking plate and $3 \%$ in another group with 
locking calcaneal plate. None of his cases had osseous infection [5]. Recent data from Zhou et al., reported a 13.5\% wound complication rate in 37 patients in the lateral extensile approach group [10]. A review article by Xiao et al., involving 2046 patients who had open reduction and internal fixation of calcaneal fracture had a wound complication rate of $13.6 \%$ [11].

In relation to operative time, it was statistically not significant $(\mathrm{p}=0.272)$ between the cases with complications and those with not. Similar finding was reported by Al-Mudhaffar et al. [4]. Smoking has been regarded as a well-known risk factor for foot and ankle surgery [12] [13] [14], and all the infected cases (n $=4)$ in our series were smokers. However, it was not statistically significant ( $\mathrm{p}=$ $0.113)$.

Comminuted fracture with difficult reduction (difficult Sanders II and Sanders III) [15] [16] and surgeons with inadequate experience required longer operating hours which contributed to higher wound complication [17].

There was no increase in wound complication in patients with concomitant fractures. Only 1 patient with a lumbar burst fracture had redness over the surgical site at 2 weeks follow up and it resolved with oral antibiotics. His wound completely healed at 6 weeks follow up (Table 3 ).

It is appropriate to delay the surgery until the presence of the wrinkle sign. Ice therapy and strict elevation is vital to allow healing of the soft tissues and to reduce swelling and pain. While waiting for the swelling to subside in majority of the cases (mean 9.2 days in our series), we often discharge the patient with Robert Jones bandage and they are advised to avoid suspending the foot at home. We will see the patient 1 to 2 days prior to the surgery day.

Gentle handling of soft tissue during raising of the lateral skin flaps to ensure good circulation is the most important factor in our opinion [18] [19]. Blade size 15 was used to make a curvilinear incision straight down to bone. Retracting the flap with a suture anchor and using the "no touch" technique minimizes soft tissue trauma.

We present in this series a lower infection rate using lateral extensile compared to others published in the literature [3] [4] [5] [9] [10] [11]. Important risk factors found in this review and other publications are used as a guide to reduce the complication rate. In recent years, newer approaches such as the sinus

Table 3. Association of concomitant fracture to wound complication.

\begin{tabular}{cccc}
\hline Injuries & Number & $\begin{array}{c}\text { Wound complication } \\
\text { at 2 weeks }\end{array}$ & $\begin{array}{c}\text { Wound complication } \\
\text { at } 6 \text { weeks }\end{array}$ \\
\hline Isolated calcaneum fracture & 26 & 5 & $1^{* *}$ \\
Bilateral calcaneum fracture & 6 & 0 & 0 \\
Spine fracture (Lumbar burst) & 2 & $1^{*}$ & 0 \\
Distal radius fracture & 1 & 0 & 0 \\
Tibia plateau fracture & 1 & 0 & 0 \\
\hline
\end{tabular}

${ }^{\star}$ Redness of surgical site, completely resolved at 6 weeks; ${ }^{*}$ Definitive wound complication at 6 weeks. 


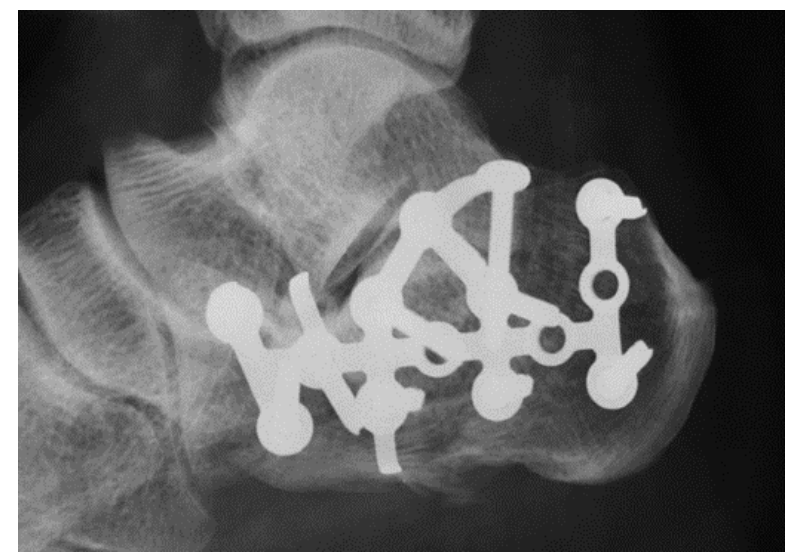

Figure 5. Post-operative radiograph of calcaneum fixation using the lateral expansile approach.

tarsi approach and minimal invasive techniques are recommended to reduce the infection rate [20] [21] [22] [23]. However these approaches may not be suitable for all types of fractures.

Poor choice of approach may lead to a mal reduced fracture and cause excessive soft tissue retraction leading to a disastrous effect on wound healing. The reliability of fracture reduction in the lateral extensile approach is constant, practical and relatively straightforward.

Based on our experience and findings, were commended to perform the surgery when soft tissue condition is good but not to delay the fracture more than 2 weeks. Surgeons should plan ahead of reduction steps to aim for shorter surgery time and minimize bleeding. Meticulous soft tissue handling throughout the surgery is a must.

Limitations of this study include the retrospective design with a small number of patients, multiple operating surgeons, incomplete wound complication description and lack of good documentation of perioperative information.

\section{Conclusion}

Intraarticular calcaneum fractures remain a difficult and challenging surgery. To reduce the complication rate, choosing the correct approach for surgery, good soft tissue condition prior to surgery, shorter operative time, meticulous soft tissue handling, proper anatomical fracture reduction, the learning curve of the surgeon and advising patients to stop smoking are all important factors for good outcome and also to reduce wound complications. In our opinion, it is a safe and appropriate approach for intraarticular calcaneum fracture reduction and internal fixation (Figure 5).

\section{Acknowledgements}

The author would like to thank the clinical research center, Hospital Raja Permaisuri Bainun, HRPB for the advice and help on statistical analysis and evaluation. Special thanks to Mr Asni Alias (former Chief Foot and Ankle Trainer, 
HRPB) for his guidance and teaching. We are grateful to Mr Manoharan (Head of Department Orthopaedic HRPB) for his support.

\section{Conflicts of Interest}

No benefits in any form have been received or will be received from a commercial party related directly or indirectly to the subject of this article. No funds were received in support of this study.

\section{References}

[1] Thermann, H., Krettek, C., Hüfner, T., Schratt, H.E., Albrecht, K. and Tscherne, H. (1998) Management of Calcaneal Fractures in Adults: Conservative versus Operative Treatment. Clinical Orthopaedics and Related Research, 353, 107-124. https://doi.org/10.1097/00003086-199808000-00013

[2] Yeap, E.J., Rao, J., Pan, C.H., Soelar, S.A. and Younger, A.S. (2016) Is Arthroscopic Assisted Percutaneous Screw Fixation as Good as Open Reduction and Internal Fixation for the Treatment of Displaced Intra-Articular Calcaneal Fractures? Foot and Ankle Surgery, 22, 164-169. https://doi.org/10.1016/j.fas.2015.06.008

[3] Koski, A., Kuokkanen, H. and Tukiainen, E. (2005) Postoperative Wound Complications after Internal Fixation of Closed Calcaneal Fractures: A Retrospective Analysis of 126 Consecutive Patients with 148 Fractures. Scandinavian Journal of Surgery, 94, 243-245. https://doi.org/10.1177/145749690509400313

[4] Al-Mudhaffar, M., Prasad, C.V. and Mofidi, A. (2000) Wound Complications Following Operative Fixation of Calcaneal Fractures. Injury, 31, 461-464. https://doi.org/10.1016/S0020-1383(00)00026-7

[5] Rak, V., Ira, D. and Masek, M. (2009) Operative Treatment of Intra-Articular Calcaneal Fractures with Calcaneal Plates and Its Complications. Indian Journal of Orthopaedics, 43, 271. https://doi.org/10.4103/0019-5413.49388

[6] Rammelt, S., Barthel, S., Biewener, A., Gavlik, J.M. and Zwipp, H. (2003) Calcaneus Fractures. Open Reduction and Internal Fixation. Zentralblatt fur Chirurgie, 128, 517-528.

[7] Herscovici Jr., D., Widmaier, J., Scaduto, J.M., Sanders, R.W. and Walling, A. (2005) Operative Treatment of Calcaneal Fractures in Elderly Patients. The Journal of Bone and Joint Surgery, 87, 1260-1264. https://doi.org/10.2106/00004623-200506000-00010

[8] Yeo, J.H., Cho, H.J. and Lee, K.B. (2015) Comparison of Two Surgical Approaches for Displaced Intra-Articular Calcaneal Fractures: Sinus Tarsi versus Extensile Lateral Approach. BMC Musculoskeletal Disorders, 16, 63. https://doi.org/10.1186/s12891-015-0519-0

[9] Folk, J.W., Starr, A.J. and Early, J.S. (1999) Early Wound Complications of Operative Treatment of Calcaneus Fractures: Analysis of 190 Fractures. Journal of Orthopaedic Trauma, 13, 369-372. https://doi.org/10.1097/00005131-199906000-00008

[10] Zhou, H.C., Yu, T., Ren, H.Y., Li, B., Chen, K., Zhao, Y.G. and Yang, Y.F. (2017) Clinical Comparison of Extensile Lateral Approach and Sinus Tarsi Approach Combined with Medial Distraction Technique for Intra-Articular Calcaneal Fractures. Orthopaedic Surgery, 9, 77-85. https://doi.org/10.1111/os.12310

[11] Yu, X., Pang, Q.J., Chen, L., Yang, C.C. and Chen, X.J. (2014) Postoperative Complications after Closed Calcaneus Fracture Treated by Open Reduction and Internal Fixation: A Review. Journal of International Medical Research, 42, 17-25. 
https://doi.org/10.1177/0300060513495626

[12] Ding, L., He, Z., Xiao, H., Chai, L. and Xue, F. (2013) Risk Factors for Postoperative Wound Complications of Calcaneal Fractures Following Plate Fixation. Foot \& Ankle International, 34, 1238-1244. https://doi.org/10.1177/1071100713484718

[13] Malay, D.S. (2011) Cigarette Smoking and Foot and Ankle Surgery. The Journal of Foot and Ankle Surgery, 50, 515-516. https://doi.org/10.1053/j.jfas.2011.08.001

[14] Zeman, P., Zeman, J., Matejka, J. and Koudela, K. (2008) Long-Term Results of Calcaneal Fracture Treatment by Open Reduction and Internal Fixation Using a Calcaneal Locking Compression Plate from an Extended Lateral Approach. Acta Chirurgiae Orthopaedicae et Traumatologiae Cechoslovaca, 75, 457-464.

[15] Backes, M., Spierings, K.E., Dingemans, S.A., Goslings, J.C., Buckley, R.E. and Schepers, T. (2017) Evaluation and Quantification of Geographical Differences in Wound Complication Rates Following the Extended Lateral Approach in Displaced Intra-Articular Calcaneal Fractures-A Systematic Review of the Literature. Injury, 48, 2329-2335. https://doi.org/10.1016/j.injury.2017.08.015

[16] Howard, J.L., Buckley, R., McCormack, R., Pate, G., Leighton, R., Petrie, D. and Galpin, R. (2003) Complications Following Management of Displaced Intra-Articular Calcaneal Fractures: A Prospective Randomized Trial Comparing Open Reduction Internal Fixation with Nonoperative Management. Journal of Orthopaedic Trauma, 17, 241-249. https://doi.org/10.1097/00005131-200304000-00001

[17] Schepers, T., Den Hartog, D., Vogels, L.M. and Van Lieshout, E.M. (2013) Extended Lateral Approach for Intra-Articular Calcaneal Fractures: An Inverse Relationship between Surgeon Experience and Wound Complications. The Journal of Foot and Ankle Surgery, 52, 167-171. https://doi.org/10.1053/j.jfas.2012.11.009

[18] Bibbo, C., Ehrlich, D.A., Nguyen, H.M., Levin, L.S. and Kovach, S.J. (2014) Low Wound Complication Rates for the Lateral Extensile Approach for Calcaneal ORIF When the Lateral Calcaneal Artery Is Patent. Foot \& Ankle International, 35, 650-656. https://doi.org/10.1177/1071100714534654

[19] Carow, J.B., Carow, J., Gueorguiev, B., Klos, K., Herren, C., Pishnamaz, M., Weber, C.D., Nebelung, S., Kim, B.S. and Knobe, M. (2018) Soft Tissue Micro-Circulation in the Healthy Hindfoot: A Cross-Sectional Study with Focus on Lateral Surgical Approaches to the Calcaneus. International Orthopaedics, 42, 2705-2713. https://doi.org/10.1007/s00264-018-4031-7

[20] Abdelazeem, A., Khedr, A., Abousayed, M., Seifeldin, A. and Khaled, S. (2014) Management of Displaced Intra-Articular Calcaneal Fractures Using the Limited Open Sinus Tarsi Approach and Fixation by Screws Only Technique. International Orthopaedics, 38, 601-606. https://doi.org/10.1007/s00264-013-2203-z

[21] Li, L.H., Guo, Y.Z., Wang, H., Sang, Q.H., Zhang, J.Z., Liu, Z. and Sun, T.S. (2016) Less Wound Complications of a Sinus Tarsi Approach Compared to an Extended Lateral Approach for the Treatment of Displaced Intraarticular Calcaneal Fracture: A Randomized Clinical Trial in 64 Patients. Medicine, 95, e4628. https://doi.org/10.1097/MD.0000000000004628

[22] Li, S. (2018) Wound and Sural Nerve Complications of the Sinus Tarsi Approach for Calcaneus Fractures. Foot \& Ankle International, 39, 1106-1112. https://doi.org/10.1177/1071100718774808

[23] Park, J. and Che, J.H. (2017) The Sinus Tarsi Approach in Displaced Intra-Articular Calcaneal Fractures. Archives of Orthopaedic and Trauma Surgery, 137, 1055-1065. https://doi.org/10.1007/s00402-017-2714-y 